\title{
Code Transformations Based on Speculative SDC Scheduling
}

\author{
Marco Lattuada and Fabrizio Ferrandi \\ Dipartimento di Elettronica, Informazione e Bioingegneria \\ Politecnico di Milano - Italy \\ Email: marco.lattuada@polimi.it fabrizio.ferrandi@polimi.it
}

\begin{abstract}
Code motion and speculations are usually exploited in the High Level Synthesis of control dominated applications to improve the performances of the synthesized designs. Selecting the transformations to be applied is not a trivial task: their effects can indeed indirectly spread across the whole design, potentially worsening the quality of the results.
\end{abstract}

In this paper we propose a code transformation flow, based on a new extension of the System of Difference Constraints (SDC) scheduling algorithm, which introduces a large number of transformations, whose profitability is guaranteed by SDC formulation. Experimental results show that the proposed technique in average reduces the execution time of control dominated applications by $37 \%$ with respect to a commercial tool without increasing the area usage.

\section{INTRODUCTION}

Programmable devices such as FPGAs can potentially offer very significant computational power, but implementing efficient solutions on them can be a hard task. One of the main obstacles is the usage of hardware description language, whose knowledge is usually a rare expertise. To overcome or at least to mitigate this issue, High Level Synthesis (HLS) [1] has been introduced. Because of the characteristics of FPGAs, HLS can quite easily produce efficient implementations of data dominated applications which are the most suitable to be implemented in hardware. On the contrary, the implementations of control dominated applications can be very inefficient, especially when compared to software implementations because of the higher frequency of general purpose processors. Nevertheless, there can still be the needing to execute these specifications in hardware. For example, they can be part of larger applications or system full hardware implementations have to be preferred to heterogeneous solutions because of the reduced data transfers. Since the data dominated parts of the applications can be implemented in a very efficient way, the control dominated portions can become the bottleneck, so they have to be optimized as well.

One of the most critical problems of optimizing a control dominated specification is the scheduling, i.e., deciding in which control step each operation is executed. Most of the scheduling techniques (e.g., [2], [3]) compute the scheduling starting from the Control-DataFlow Graph (CDFG) [4], a graph based description which represents the control and the data dependences of the analyzed specification. The structure of the $C D F G$ can heavily determine the scheduling results, even if exact methods (e.g., [2]) are used. Indeed, scheduling algorithms can produce very different results (better or worse) if they start from a modified version of the $C D F G$, obtained by applying code motion or speculation. Applying this type of transformation is not a trivial task since evaluating the overall effects of a single or of a set of transformations is not easy. State of the art methodologies (e.g., [3], [5]) typically exploit heuristics and consider only a limited set of possible transformations, potentially limiting the benefits of this type of approach.

In this paper a methodology flow based on an exact method for explicitly transforming a $C D F G$ to improve the scheduling solution is proposed. The main contributions of this work are:

- it extends the SDC scheduling algorithm formulation [2] to allow implicit global code motion;

- it exploits the results of the modified SDC scheduling algorithm to perform explicit code motion.

The rest of this paper is organized as follows: Section II describes the problem addressed by the proposed methodology which is described in Section III. Section IV presents the experimental evaluation of the proposed flow, then Section V compares the proposed methodology with state of the art techniques and finally Section VI draws the conclusion of this work and presents the possible future extensions.

\section{Problem Definition}

The proposed methodology aims at modifying the $C D F G$ to improve the scheduling solution in terms of overall latency of the application. $C D F G$ is a directed graph $G_{C D F G}=$ $\left(V_{o p} \cup V_{b b}, E_{o p} \cup E_{b b}\right)$ where each vertex $v_{o p} \in V_{o p}$ corresponds to an operation, each vertex $v_{b b} \in V_{b b}$ corresponds to a basic block, each edge $e_{o p} \in E_{o p}$ represents a data dependences between two operations and each edge $e_{b b} \in E_{b b}$ represents the control dependences between two basic blocks. Figure 1b shows an example of $C D F G$, extracted from the code of Figure 1a. In the following it is assumed that there are only two available multipliers, the division $v_{3}$ takes 3 cycles, all the other operations take 1 cycle. For the sake of simplicity operations chaining is not considered in the presented example, even if it is fully supported in the proposed methodology.

$C D F G$ is modified by means of explicit code motions: operations are moved from their current basic block to other basic block preserving the semantic of the application. The code motion can be applied directly on the $C D F G$ or on the code from which it has been generated. Not all the possible code motions are considered: operations cannot be moved from a loop to another one, nor can be moved from after a loop to before a loop. The proposed methodology assumes that the 


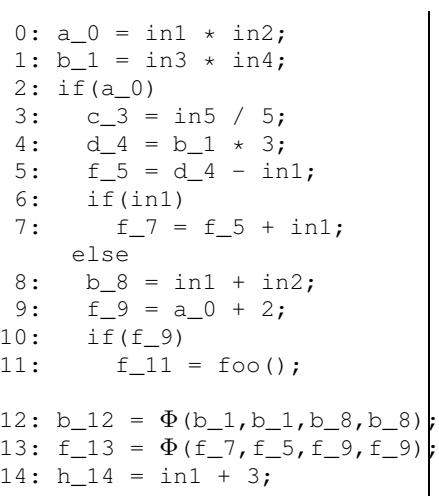

(a) Starting Code

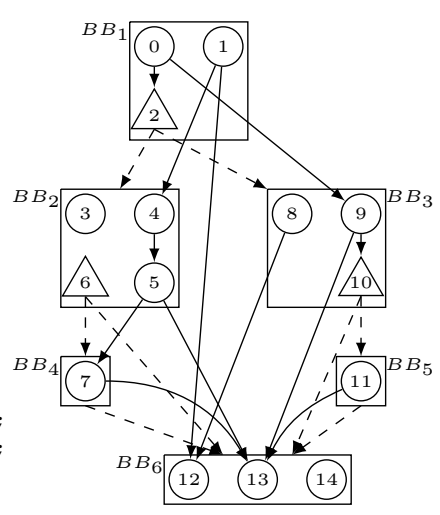

(b) Starting CDFG

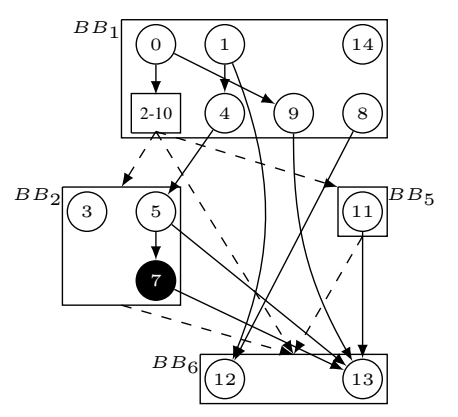

(f) Final CDFG

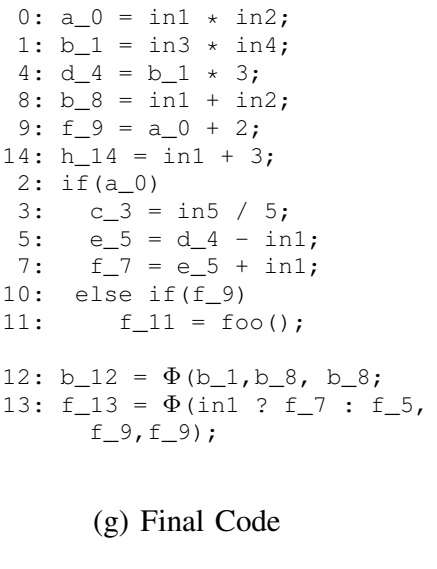

Fig. 1: An example of application of the proposed methodology.

analyzed specification is already in Static Single Assignment form [6], condition which is already satisfied in most of the compilers and high level synthesis tools. In this form, each variable must be assigned exactly once, so a new version of a variable is created for each its assignment (e.g., f_5, f_7, $\left.\mathrm{f} \_9, \mathrm{f} \_11\right)$. Variables used in right side of statement are renamed so that the most recent definition of a variable is used. If multiple definitions of a same variable reach a basic block (e.g., f_5, f_7, f_9, f_11 reach $\left.B B_{6}\right)$, they are merged by means of a new artificial definition $\Phi$, which creates a new version of the variable (e.g., f_13 $f_{-} \Phi\left(f_{-} 7, f_{-5}, f_{-} 9, f_{-} 9\right)$ ).

Code motions can be classified in:

- Non-speculative: the operation is moved between two basic blocks that are executed in exactly the same traces. This transformation is always safe, but it still has to be applied in a conservative way since it can increase the overall latency and the area of the solution. An example of Non-speculative code motion in example of Figure 1 is $v_{14}$ to $B B_{1}$

- Speculative: an operation $v_{i}$ of basic block $B B_{a}$ is moved in a $B B_{b}$ that is executed in a superset of the traces containing $B B_{a}$, i.e., it is anticipated with respect to the conditional construct which controls its execution. These transformations can be applied only if the operation does

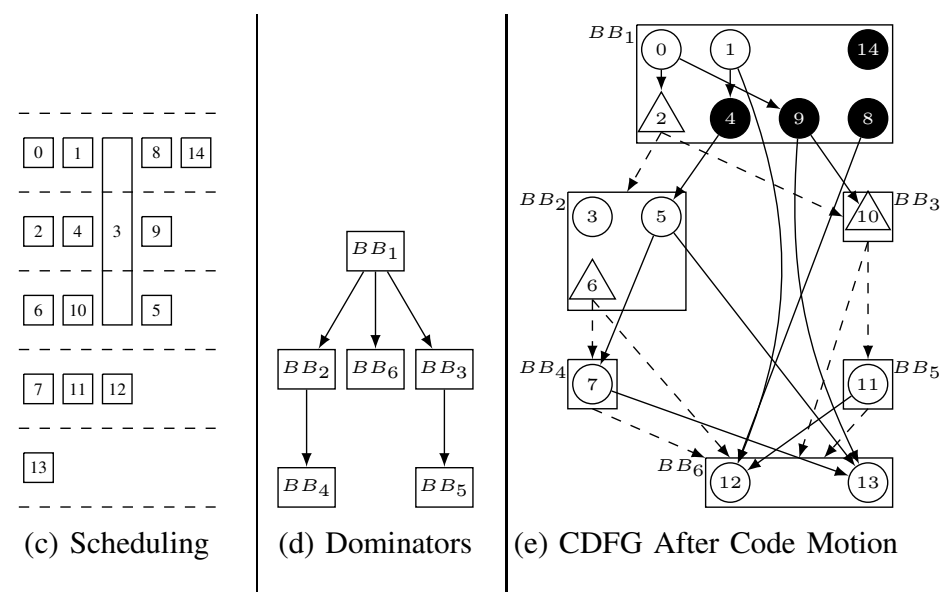

not modify memory status. An example of Speculative code motion in example of Figure 1 is $v_{4}$ in $B B_{1}$. Use of speculated operations instead of operations controlled by guards like in [2] can improve performance results (they have not to wait for the guard), but they can increase the area usage.

In the next section how to solve the problem of identifying only profitable transformations will be presented.

\section{PROPOSED Methodology}

The proposed methodology flow is composed of three steps:

1) Speculative SDC scheduling: operations are scheduled with SDC scheduling algorithm extended to allow code motion.

2) Code Motion: code motions suggested by SDC scheduling results are applied to the analyzed $C D F G$.

3) Transformations: $C D F G$ is transformed by means of heuristics to further improve overall performances.

In the following each step of the flow will be detailed.

\section{A. Speculative SDC Scheduling}

This section describes Speculative SDC scheduling, an extension of SDC scheduling algorithm [2] aimed at supporting code motion. Since code motion has to be allowed only intra loops, Speculative SDC scheduling is independently applied on each loop (the whole function is the most external loop). Its formulation uses the following variables and functions:

- $O P_{k}$ : the set of operations contained in loop $l_{k}$ and not contained in any loop nested in $l_{k}$.

- $S E$ : the set of operations which cannot be speculated because of side effects (e.g., store operations) or because they are conditional constructs (e.g., if).

- $\operatorname{Cond}\left(v_{i}\right)$ : the conditional construct which controls the execution of an operation [7].

- $L v_{i}$ : the cycle latency of $v_{i}$.

- $T$ : the clock period.

- $D\left(v_{i}, v_{j}\right)$ : estimation of the delay of the longest critical combinational path between $v_{i}$ and $v_{j}$. The estimations are obtained by applying the same approach adopted in 
[2] (i.e., adding the estimated delay of all the operations and of all the interconnections of the path).

$\forall l_{k}, \forall v_{i} \in O P_{k}, \forall t \in\left[0, L v_{i}\right]: s v_{t}\left(v_{i}\right) \in \mathbb{N} \cup\{0\}$ is the variable specifying the control step in which the stage of an operation is scheduled. $s v_{\text {beg }}(v)$ and $s v_{e n d}(v)$ are defined as follows: $s v_{b e g}(v) \equiv s v_{0}(v), s v_{e n d}(v) \equiv s v_{L v}(v)$. Moreover, for each basic block $B B_{b}$ an artificial node $\operatorname{ssnk}\left(B B_{b}\right)$ is added representing its ending. The constraints are:

1) $\forall v_{i} \in O P_{k} \mid L v_{i} \geq 1, \forall t \in\left[1, L v_{i}\right]: s v_{t}\left(v_{i}\right)=$ $s v_{t-1}\left(v_{i}\right)+1$ : consecutive stages of a multicycle operation must be executed in consecutive control steps (e.g., the three stages of $v_{3}$ have to be executed in consecutive control steps).

2) $\forall v_{i} \in O P_{k} \mid v_{i} \in S E \wedge \operatorname{Cond}\left(v_{i}\right) \neq \varnothing$ : $s v_{\text {end }}\left(\operatorname{Cond}\left(v_{i}\right)\right)-s v_{\text {begin }}(v) \leq-1$ : operations which cannot be speculated have to be scheduled after the conditional construct which controls them (e.g., $v_{11}$ must be scheduled after $v_{10}$ ).

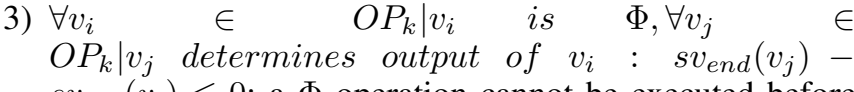
$s v_{\text {beg }}\left(v_{i}\right) \leq 0$ : a $\Phi$ operation cannot be executed before the conditional construct which controls which variable version has to be selected (e.g., $v_{12}$ and $v_{13}$ cannot be scheduled before $v_{6}$ and $\left.v_{10}\right)$.

4) $\forall v_{i} \in O P_{k}: s v_{\text {end }}\left(v_{i}\right)-s v_{\text {end }}\left(B B_{b}\right) \leq 0$ (where $B B_{b}$ is the basic block to which $v_{i}$ belongs): a basic block does not end before the ending of any its operation (e.g., $B B_{3}$ does not end before $v_{8}, v_{9}$ and $v_{10}$ end).

5) $\forall e\left(v_{i}, v_{j}\right) \in E_{d}: s v_{\text {end }}\left(v_{i}\right)-s v_{\text {beg }}\left(v_{j}\right) \leq 0$ : data dependencies between operations must be guaranteed (e.g., $v_{4}$ cannot start before the end of $v_{5}$ ).

6) $\forall v_{i}, v_{j} \mid$ exists a data dependence path $\left(v_{i} \rightarrow v_{j}\right)$ in $C D F G: s v_{b e g}\left(v_{i}\right)-s v_{b e g}\left(v_{j}\right) \leq-\left(\left[D\left(v_{i}, v_{j}\right) / T\right]-1\right)$ : a pair of operations whose longest critical combinational path is larger than clock period cannot be chained (e.g., $v_{2}$ cannot start in the same control step of $v_{0}$ ).

7) resource sharing: for each execution trace inside the $C D F G$ and for each limited resource $r$, a linear order between operations $V_{r}$ mapped on resources of type $r$ is built. If $N_{r}$ is the number of resources $r$, the $i^{t h}$ and the $i^{t h+N_{r}}$ operations of the linear order cannot be executed in the same control step: $s v_{b e g}\left(v_{i}\right)-s v_{b e g}\left(v_{i+N_{r}}\right) \leq-1$ is added to the formulation (e.g., given the linear order of operations mapped on multiplier $v_{0}-v_{1}-v_{4}$, since there are only two available multipliers, $v_{4}$ cannot start in the same cycle of $v_{0}$ ).

The last type of constraints do not avoid the simultaneous scheduling of multiple speculated operations which can require more resources than the available. Instead of adding a set of linear orders between these operations, which introduce a set of unnecessary constraints when these operations are actually not speculated, it is admitted that the solutions produced by SDC are actually not implementable. The following steps of the methodology will transform these solutions so that they will respect resource constraints. The first significant difference with respect to [2] is the absence of ssrc nodes, i.e., nodes that represent beginning of basic blocks, and of the control dependence constraints, since they prevent speculation of operations. Since speculation of $S E$ operations has still to be avoided, the constraints of type 2 are added. The second

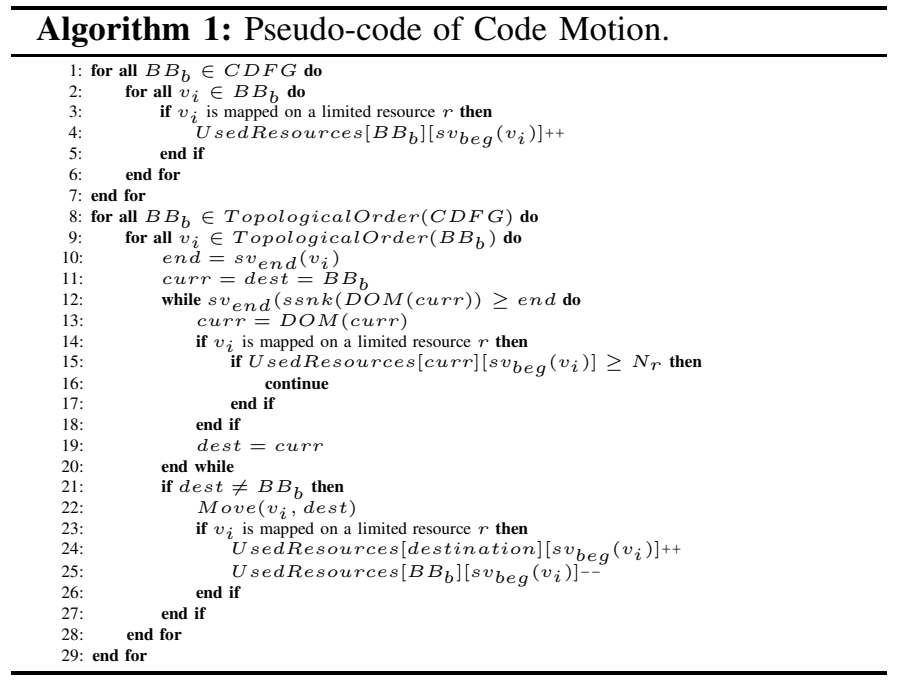

main difference is the introduction of constraints of type 3 to model the implicit dependences between a $\Phi$ operation and the conditional constructs which determine its outcome. Finally, the last significant difference is the objective function used to minimize the overall latency of the solution. In [2] the overall latency is approximated by an expression based on the beginning and the ending of each basic block. Since the beginning of basic blocks cannot be specified in Speculative SDC formulation, a different objective function is adopted:

$$
\min \sum_{B B_{i} \in l_{k}} s v_{e n d}\left(\operatorname{ssnk}\left(B B_{i}\right)\right)
$$

By minimizing this objective function, all the longest paths from the header of the loop (its first basic block) to all its basic blocks are minimized at the same time. On the contrary other paths can be only partially optimized (e.g., $B B_{1}-B B_{3}-B B_{6}$ ), so also the proposed objective function is an approximation, but differently from the one proposed in [2] does not require path profiling information.

The Speculative SDC scheduling solution of $C D F G$ of Figure $1 \mathrm{~b}$ is shown in Figure 1c.

\section{B. Code Motion}

In this step, the results of the Speculative SDC scheduling algorithm are used to identify which operations can be moved from a basic block to another improving the overall performance of the scheduling solution. The basic block to which each operation has to be moved is identified by analyzing the dominator tree [8] built starting from the $C D F G$. A basic block $B B_{d}$ dominates basic block $B B_{i}$ if every path from the entry of $C D F G$ to $B B_{i}$ go through $B B_{d}$. A basic block $B B_{d}$ immediately dominates $B B_{i}$ if dominates $B B_{i}$ and there is not any $B B_{h}$ such that $B B_{d}$ dominates $B B_{h}$ and $B B_{h}$ dominates $B B_{i}$. The immediate dominator relationship is described by dominator tree. Dominator tree of example of Figure $1 \mathrm{~b}$ is shown in Figure 1d. Algorithm 1 describes in details how code motion is performed. All the operations of the $C D F G$ are analyzed in topological order (loops of lines 8 and 9). An operation can be moved in a dominator if it does not end after the end of the dominator (line 12). The 
algorithm goes up through the immediate dominator chain as long as it encounters basic blocks where it can move the operation (line 13). Figure 1e shows the $C D F G$ after application of code motion: black nodes are moved operations. For example $v_{14}$ is moved in $B B_{1}$ (Non-speculative code motion) since $s v_{\text {end }}\left(v_{14}\right) \leq s v_{\text {end }}\left(\operatorname{ssnk}\left(B B_{1}\right)\right)(1 \leq 2), v_{4}$ is moved in $B B_{1}$ (Speculative code motion) since $s v_{\text {end }}\left(v_{4}\right) \leq$ $s v_{\text {end }}\left(\operatorname{ssnk}\left(B B_{1}\right)\right)(2 \leq 3), v_{3}$ cannot be moved in $B B_{1}$ since $s v_{\text {end }}\left(v_{3}\right) \not \leq s v_{\text {end }}\left(\operatorname{ssnk}\left(B B_{1}\right)(3 \not 2)\right.$. By considering only dominators as possible candidate destination of code motion, it is guaranteed that the execution of the moved operation is not removed by any execution trace, but it can eventually be added to further execution traces (Speculative code motion). The check of the ending time of the destination basic block guarantees that input data of the operation will be ready in that basic block and that the ending of this will be not postponed by the moved operation.

In case of operations mapped on limited resources (line 14), it has to be checked that the ending of the destination basic block is not postponed because of resource contention in a particular control step (line 15). To perform this check, it is necessary to have information about the utilization of limited resources of operations of each basic block in each control step (lines 1-7). When the actual destination has been finally identified, code motion can be actually performed. Since the methodology assumes that the intermediate representation is in SSA form, no further change (e.g., fixing of variables or of $\Phi$ instructions) is required.

It is worth noting that SDC scheduling algorithm applied on the transformed $C D F G$ can produce a solution different from Speculative SDC solution because not all the code motions suggested by Speculative SDC can actually be applied. There are two main reasons for which the code motion of an operation is not performed: it would violate a resource constraint or it would require to divide a multi-cycle operation among different basic blocks. For example $v_{3}$ should be distributed between $B B_{1}$, and $B B_{2}$.

\section{Code Transformations}

Since the $C D F G$ produced in the previous step does not correspond exactly to the Speculative SDC solution, there can still be the possibility of optimizing it by applying transformations not included in the optimal solution computed by Speculative SDC scheduling. Some of these transformations can be applied directly on the modified $C D F G$ by means of heuristics, others can be applied only after that the structure of the $C D F G$ has been cleaned.

Algorithm 2 shows the order in which the different transformations are applied on each basic block:

1) Local Code Motion (lines 2-12): each operation is analyzed checking if it can be moved in one of the dominator of the current basic block. Profitability of a code motion is not evaluated anymore by considering Speculative SDC solution, but analyzing the relative scheduling of the operations in the destination basic block: an operation $v_{i}$ can be moved in dominator $B B_{d o m}$ if its input operands are all available during execution of $B B_{d o m}$ and if it does not increase the overall latency of $B B_{d o m}$. Indeed, this type of increment has to be avoided since it would

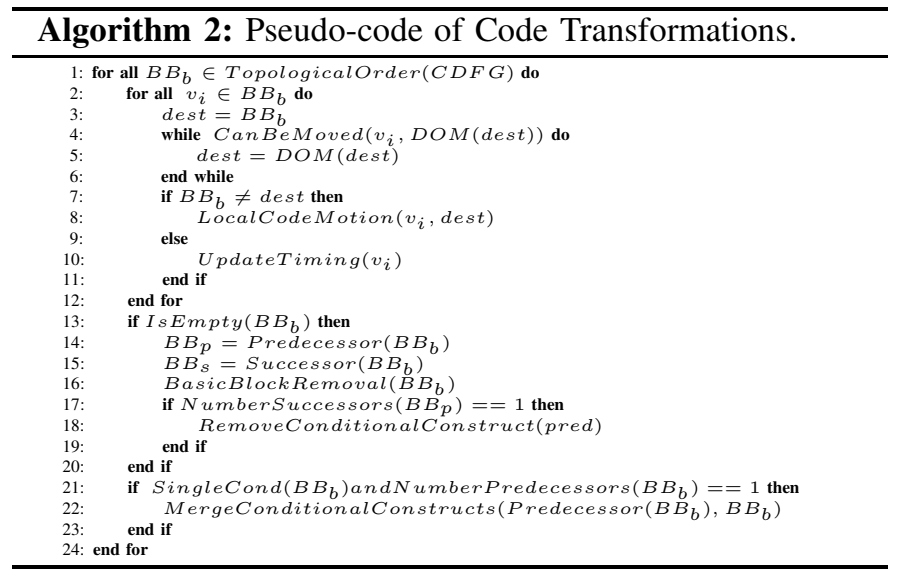

increase the overall delay of the solution. In the $C D F G$ of Figure 1e $v_{7}$ can be moved in $B B_{2}$ since it does not increase its latency (3). Note that this code motion is not suggested by Speculative SDC because of the ending time of $B B_{2}$ : according to Speculative SDC solution $v_{7}$ should be scheduled in 4 while the ending time of $B B_{2}$ is 3 , but this can be obtained only by splitting execution of $v_{3}$ on $B B_{1}$ and $B B_{2}$.

2) Basic Block Removal (line 16): empty basic blocks are removed. In example of Figure 1e, $B B_{4}$ can be removed since it becomes empty after the moving of $v_{7}$.

3) Remove Conditional Construct (line 18): after removing an empty basic block, it is possible that all the targets of a conditional construct become the same: in this case, the corresponding $\Phi$ instructions are fixed and then the conditional construct is removed. After the removal of $B B_{4}, v_{6}$ targets only $B B_{6}$, so $v_{6}$ is removed and $v_{13}$ is transformed in $f_{-13}=\Phi($ inl ? f_7 : f_5, f_9, f_9).

4) Merge Conditional Constructs (line 22): if a basic block $B B_{b}$ is composed only of a conditional construct and it has only one predecessor $B B_{p}, \Phi$ instructions of successors of $B B_{b}$ are fixed, the conditional construct of $B B_{b}$ is merged with the conditional construct at the end of $B B_{p}$ and $B B_{b}$ is removed. In example of Figure 1e, $B B_{3}$ is composed only of $v_{10}$, so $v_{2}$ and $v_{10}$ can be merged and $B B_{3}$ can be removed.

Figure 1f shows the $C D F G$ after that all the presented transformations have been applied while the corresponding code is presented in Figure 1g. In $C D F G$ of Figure $1 \mathrm{~b}$ four execution paths can be identified whose delays in terms of clock cycles are: 7,6,5,6. After that the proposed methodology is applied, in $C D F G$ of Figure 1f their delays become: 6,6,3,4.

\section{Complexity of the proposed methodology}

The complexity of solving a System of Difference Constraints is $\Theta(n \cdot m)$ where $n$ is the number of variables and $m$ is the number of constraints. Since the Speculative SDC and the original formulation of SDC share all the variables and most of the constraints (types 1,4,6,7), their complexity is comparable. In Speculative SDC formulation there are also constraints of type 2,3, but there are not constraints for ssrc and the number of added constraints of type 5 is smaller (since inter loops dependences have not to be considered). In 
the worst case, both for original formulation of SDC and for Speculative SDC, the number of variables is $O\left(\left|O P_{k}\right|\right)$ and the number of constraints is $O\left(\left|O P_{k}\right|^{2}\right)$, so the complexity of the first step of the proposed methodology is $O\left(\left|O P_{k}\right|^{3}\right)$. The complexity of the second step is $O\left(\left|O P_{k}\right|\right.$ ) (loops of lines 8 and 9 of Algorithm 1) while the complexity of the Transformations step corresponds to the complexity of Algorithm 2 which is $O\left(\left|B B_{b}\right|\right)$. Since the two last steps have smaller complexity than the Speculative SDC, the overall complexity of the methodology is $O\left(\left|O P_{k}\right|^{3}\right)$.

\section{EXPERIMENTAL RESULTS}

To verify the effectiveness of the proposed methodology, the results of four High Level Synthesis flows have been compared:

(1) Commercial Tool, which implements SDC scheduling algorithm and which supports Xilinx FPGAs; this tool supports code motion but only for code of pipelined loops which are not contained in the considered benchmark suite.

(2) LegUp 3.0 [9], an open source publicly available High Level Synthesis Tool developed at University of Toronto which already implements SDC scheduling algorithm and which supports Altera FPGAs.

(3) PandA 0.9.3 [10], an open source, publicly available framework for High Level Synthesis developed at Politecnico di Milano, extended with the SDC scheduling algorithm presented in [2]. In the following it will be shown how the results obtained with this implementation are in average quite similar to the ones produced by (1), so that this implementation can be considered a good golden reference.

(4) PandA 0.9.3 [10] extended with the proposed methodology. Intermediate representation adopted in PandA is already in SSA form, so implementation of the proposed methodology only requires to implement Speculative SDC scheduling and the proposed code transformations. This tool has been chosen as starting point since it supports both Altera and Xilinx FPGAs allowing to verify the effective generality of the proposed methodology.

For the sake of fairness, the comparison with [5] has not been performed since it does not support operations chaining.

Two platforms have been considered in the experimental evaluation:

- the Xilinx Zynq-7000 xc7z0 (not supported by LegUp) with a target frequency of $66.66 \mathrm{MHz}$ (same default experimental setup of (1)). Final synthesis is performed by means of Xilinx Vivado [11].

- the Altera Cyclone II EP2C70F896C6 (not supported by commercial tool) with the target frequency of $66.66 \mathrm{M} \mathrm{Hz}$ (same default experimental setup of (2)). Final synthesis is performed by means of Altera Quartus II [12].

The benchmarks adopted to perform the experimental evaluation are the CHStone suite [13]. CHStone suite is a set of 12 benchmarks, explicitly collected for the evaluation of High Level Synthesis flows, which aim at representing all the possible scenarios which have to be addressed by an High Level Synthesis tool. In particular, this suite contains both data dominated applications (aes, blowfish, jpeg, mpeg2, sha) and control oriented applications (adpcm, dfadd, dfdiv, $d f m u l, d f \sin$, gsm, mips). For the sake of brevity, their detailed characteristics (e.g., the number of loops, the number of conditional constructs, the number of arithmetic operations, etc.) have not been reported, but they can been found in [13]

PandA can generate two different hardware versions of divisor according to which division algorithm is selected. The proposed methodology has been applied on both the implementations, so that two different sets of results are reported for (3) and (4) on the benchmarks which contain division operations (i.e., $d f d i v$ and $d f \sin$ ).

Left part of Table I reports the synthesis time results after place and route obtained by (1), (3), and (4) for Zynq-7000. The results of (1) on blowfish and mpeg 2 have not been reported (N/A) since the cosimulations fail. SDC scheduling implementations of (1) and (3) can be considered quite equivalent, even if there are significant differences in the results on some benchmarks, since these are not caused by SDC scheduling implementation. In particular (1) obtains better results on mips thanks to the implementation of Rotation Scheduling. On the contrary, (3) obtains better results on $d f d i v$ and $d f \sin$ thanks to the implementation of a better division algorithm. Speculative SDC scheduling does not introduce significant benefits on data dominated applications (i.e., aes, blowfish, jpeg, mpeg2, $s h a)$ as expected. On the contrary, on most of the control dominated applications (i.e., adpcm, $d f a d d, d f d i v, d f m u l, d f s i n$, gsm, mips) the gain is very significant (up to $39 \%$ on dfadd when compared to (3) and up to $46 \%$ on dfmul when compared to (1). The average gain of (4) with respect to (1) on control dominated application is instead $37 \%$.

Table II reports the synthesis area results after place and route obtained by (1), (3), and (4) targeting Zynq-7000. The produced solutions have similar resource usage in terms of Slices, LUTs, and BRAMs while the number of registers used in (3) and (4) because register allocation has not been fully optimized as in (1). The only significant difference in terms of number of BRAMs is in the synthesis of adpcm benchmark and is caused by too conservative alias analysis which prevents the application of some memory optimizations. The number of used DSPs is almost the same for all the benchmarks but $d f d i v(2)$ and $d f \sin (2)$ because of the different implemented division algorithm. The solutions produced by (3) and (4) have instead very similar results for all the resource usage metrics: the obtained benefits in terms of clock cycles by exploiting Speculative SDC scheduling have not to be paid in terms of increment of area.

Right part of Table I reports the synthesis time results after place and route obtained by (2) (results have been extracted from [9]), (3), and (4) for Cyclone II. Results in terms of cycles obtained by (3) cannot be directly compared with other tool since (2) uses a different memory model and implements different optimizations with respect to (3). The differences in terms of clock cycles in the solutions produced by (3) and (4) are similar to the ones already analyzed for Zynq-7000. (3) and (4) produce solutions with similar clock cycles latencies for data dominated applications (aes, blowfish, jpeg, mpeg2, sha), while there are significant advantages in exploiting Speculative SDC scheduling on control dominated applications (adpcm, $d f a d d, d f d i v, d f m u l, d f \sin$, gsm, mips). With respect to results 
TABLE I: Synthesis time results after place and route. (1) are the results obtained with Commercial Tool, (2) are the results obtained with LegUp, (3) and (4) are the results obtained with SDC scheduling and Speculative SDC scheduling implemented in PandA.

\begin{tabular}{|c|c|c|c|c|c|c|c|c|c|c|c|c|c|c|}
\hline & \multicolumn{7}{|c|}{ Zynq-7000 } & \multicolumn{7}{|c|}{ Cyclone II } \\
\hline & \multicolumn{3}{|c|}{ Clock Frequency } & \multicolumn{4}{|c|}{ Cycles } & \multicolumn{3}{|c|}{ Clock Frequency } & \multicolumn{4}{|c|}{ Cycles } \\
\hline & (1) & (3) & (4) & (1) & (3) & (4) & (3)-(4) & (2) & (3) & (4) & (2) & (3) & (4) & (3)-(4) \\
\hline adpcm & 34.3 & 67.3 & 67.9 & 23,075 & 17,627 & 15,297 & $13 \%$ & 45.7 & 71.1 & 66.6 & 36,795 & 19,727 & 17.347 & $12 \%$ \\
\hline dfadd & 72.0 & 83.9 & 67.2 & 383 & 355 & 219 & $39 \%$ & 124.0 & 77.6 & 70.7 & 2,330 & 380 & 289 & $24 \%$ \\
\hline $\operatorname{dfdiv}(1)$ & 817 & 69.7 & 67.6 & 1017 & 1,039 & 967 & $7 \%$ & 747 & 73.5 & 73.6 & & 1,995 & 1,821 & $9 \%$ \\
\hline $\operatorname{dfdiv}(2)$ & 81.1 & 68.1 & 64.8 & 1,911 & 762 & 547 & $16 \%$ & 14.1 & 70.6 & 69.1 & 2,14 & 910 & 810 & $11 \%$ \\
\hline dfmul & 60.7 & 74.7 & 71.8 & 196 & 149 & 105 & $28 \%$ & 85.6 & 80.6 & 76.1 & 347 & 173 & 141 & $12 \%$ \\
\hline $\mathrm{df} \sin (1)$ & 525 & 68.1 & 66.0 & 48226 & 30,161 & 26,466 & $12 \%$ & 626 & 71.9 & 70.5 & 67466 & 56,789 & 47,643 & $16 \%$ \\
\hline $\mathrm{dfsin}(2)$ & 52.5 & 68.0 & 65.5 & 48,226 & 21,182 & 17,121 & $19 \%$ & 62.6 & 71.0 & 68.5 & $6 /, 466$ & 34,658 & 26,079 & $25 \%$ \\
\hline gsm & 66.4 & 67.0 & 66.2 & 3,397 & 2,775 & 2,361 & $15 \%$ & 58.9 & 69.6 & 69.0 & 6,656 & 2,935 & 2,521 & $14 \%$ \\
\hline mips & 77.6 & 73.0 & 74.7 & 2,489 & 3,413 & 3,040 & $11 \%$ & 90.0 & 68.4 & 67.7 & 6,443 & 3,413 & 3,040 & $11 \%$ \\
\hline aes & 89.2 & 80.2 & $\overline{74.1}$ & 2,973 & 3,188 & 3,112 & $3 \%$ & 60.7 & 993.1 & 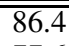 & 14,022 & 3,332 & 3,256 & $3 \%$ \\
\hline blowfish & N/A & 83.8 & 83.5 & N/A & 89,679 & 89,549 & $1 \%$ & 65.4 & 73.0 & 77.6 & 209,866 & 108,415 & 108,285 & $0 \%$ \\
\hline jpeg & 69,0 & 67.0 & 66.9 & 468,011 & 471,173 & 465,261 & $1 \%$ & 47.0 & 72.9 & 72.5 & $5,861,516$ & 490,996 & 485,084 & $1 \%$ \\
\hline mpeg2 & N/A & 93.8 & 70.4 & N/A & 4,221 & 4,214 & $0 \%$ & 91.7 & 88.0 & 78.9 & 8,578 & 4,228 & 4,225 & $0 \%$ \\
\hline sha & 97.9 & 83.1 & 87.2 & 111,043 & 113,329 & 113,324 & $0 \%$ & 86.9 & 77.2 & 75.8 & 247,738 & 123,611 & 123,606 & $0 \%$ \\
\hline
\end{tabular}

TABLE II: Synthesis area results after place and route for Zynq-7000. (1) are the results obtained with Commercial Tool, (3) and (4) are the results obtained with SDC scheduling and Speculative SDC scheduling implemented in PandA.

\begin{tabular}{|c|c|c|c|c|c|c|c|c|c|c|c|c|c|c|c|}
\hline & \multicolumn{3}{|c|}{ Slice } & \multicolumn{3}{|c|}{ LUTs } & \multicolumn{3}{|c|}{ Registers } & \multicolumn{3}{|c|}{ DSPs } & \multicolumn{3}{|c|}{ BRAMs } \\
\hline & (1) & (3) & (4) & (1) & (3) & (4) & (1) & (3) & (4) & (1) & (3) & (4) & (1) & (3) & (4) \\
\hline adpcm & 2,773 & 1,820 & 1,810 & 10,358 & 5,399 & 5,268 & 3,488 & 3,920 & 3,533 & 46 & 44 & 42 & 13 & 29 & 29 \\
\hline dfadd & 913 & 962 & 805 & 2,790 & 2,411 & 2,254 & 1,544 & 1,730 & 1,018 & 0 & 0 & 0 & 0 & 0 & 0 \\
\hline $\operatorname{dfdiv}(1)$ & & 2,372 & 2,334 & & 6,858 & 6,779 & & 4,815 & 4,526 & & 18 & 18 & & 0 & 0 \\
\hline $\operatorname{dfdiv}(2)$ & 1,288 & 1,756 & 1,661 & 4,074 & 4,957 & 4,914 & 2,726 & 3,352 & 2,918 & 24 & 66 & 66 & 0 & 1 & 1 \\
\hline dfmul & 729 & 541 & 466 & 2,433 & 1,540 & 1,341 & 1,143 & 882 & 632 & 16 & 10 & 10 & 0 & 0 & 0 \\
\hline $\mathrm{df} \sin (1)$ & & 4,680 & 4,785 & & 14,782 & 15,545 & & 10,801 & 8,549 & & 41 & 41 & & 4 & 4 \\
\hline $\mathrm{df} \sin (2)$ & 3,428 & 4,249 & 4,229 & 11,491 & 13,044 & 13,813 & 6,041 & 9,352 & 6,863 & 43 & 89 & 89 & 4 & 5 & 5 \\
\hline gsm & 1,307 & 1,441 & 1,416 & 4,569 & 4,004 & 4,099 & 1,391 & 3,221 & 3,226 & 40 & 31 & 31 & 9 & 5 & 5 \\
\hline mips & 429 & 535 & 497 & 1,359 & 1,795 & 1,728 & 511 & 644 & 669 & 8 & 8 & 8 & 4 & 4 & 4 \\
\hline$\overline{\text { aes }}$ & $\bar{~} 1,188$ & 1,355 & 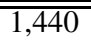 & $3,3,625$ & 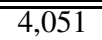 & 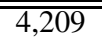 & $\bar{~} 1,888$ & 2,418 & 2,367 & $\overline{\overline{6}}$ & $\overline{0}$ & $\overline{0}$ & $\overline{13}$ & $\overline{10}$ & $\overline{10}$ \\
\hline blowfish & N/A & 876 & 838 & N/A & 2,101 & 2,217 & N/A & 2,155 & 2,144 & N/A & 0 & 0 & N/A & 22 & 22 \\
\hline jpeg & 5,870 & 4,521 & 4,593 & 18,347 & 14,291 & 13,904 & 9,683 & 10,072 & 9,756 & 14 & 15 & 9 & 55 & 59 & 59 \\
\hline mpeg2 & N/A & 645 & 621 & N/A & 1,986 & 1,887 & N/A & 1,432 & 1,194 & N/A & 0 & 0 & N/A & 2 & 2 \\
\hline sha & 647 & 735 & 720 & 2,180 & 2,140 & 2,086 & 1,356 & 2,216 & 2,214 & 0 & 0 & 0 & 20 & 12 & 12 \\
\hline
\end{tabular}

TABLE III: Synthesis area results after place and route for Cyclone II. (2) are the results obtained with LegUp, (3) and (4) are the results obtained with SDC scheduling and Speculative SDC scheduling implemented in PandA.

\begin{tabular}{|l||ccc|ccc|ccc|}
\hline \multicolumn{1}{|c||}{} & \multicolumn{3}{c|}{ LEs } & \multicolumn{3}{c|}{ Multibits } & \multicolumn{3}{c|}{ Mults } \\
& (2) & (3) & (4) & (2) & (3) & (4) & (2) & (3) & (4) \\
\hline adpcm & 22,605 & 9,819 & 10,288 & 29,120 & 13,202 & 13,302 & 300 & 120 & 88 \\
dfadd & 8,881 & 6,432 & 5,544 & 17,120 & 10,112 & 10,112 & 0 & 0 & 0 \\
dfdiv(1) & 20,159 & 11,298 & 10,356 & 12,416 & 5,054 & 5,054 & 62 & 48 & 48 \\
dfdiv(2) & & 8,096 & 7,858 & 10252 & 102 \\
dfmul & 4,861 & 3,133 & 3,011 & 12,032 & 5,120 & 5,120 & 32 & 31 & 31 \\
dfsin(1) & 39,933 & 21,484 & 20,477 & 12,864 & 6,602 & 6,602 & 100 & 70 & 85 \\
dfsin(2) & 18,415 & 17,375 & 12,864 & 7,900 & 7,900 & & 124 & 139 \\
gsm & 19,131 & 6,164 & 6,216 & 11,168 & 8,992 & 8,992 & 70 & 50 & 48 \\
mips & 4,479 & 2,559 & 2,559 & 4,480 & 4,480 & 4,480 & 8 & 16 & 16 \\
\hline \hline aes & 28,490 & 3,066 & 3,117 & 38,336 & 32,526 & 32,526 & 0 & 0 & 0 \\
blowfish & 15,064 & 3,802 & 3,933 & 150,816 & 150,528 & 150,528 & 0 & 0 & 0 \\
jpeg & 46,224 & 16,121 & 15,593 & 253,936 & 438,674 & 437,394 & 172 & 134 & 138 \\
mpeg2 & 13,238 & 2,351 & 2,328 & 34,752 & 32,768 & 32,768 & 0 & 0 & 0 \\
sha & 12,483 & 3,248 & 3,182 & 134,368 & 117,760 & 117,760 & 0 & 0 & 0 \\
\hline
\end{tabular}


obtained on Zynq-7000, the gain provided by Speculative SDC scheduling is less significant. The smaller speed of Cyclone II limits the depth of the chains of operations which can be scheduleded in a single clock cycle and so limits the number of profitful code motion. Moreover, results about maximum frequency obtained by (4) show that there is always a significant positive slack. This is caused by a conservative timing model adopted by PandA during High Level Synthesis for Cyclone II which prevents some feasible advantageous code motions and does not allow to obtain the best possible results. Finally, as in case of Zynq-7000, the area results obtained when targeting Cyclone II, which are reported in Table III, show that the gain in terms of cycles of SDC scheduling solutions does not imply an increase of resource usage.

\section{RELATED WORK}

The algorithms which have been proposed to solve the problem of scheduling in High Level Synthesis can be roughly classified in two categories. The former are oriented at optimizing data dominated applications and in particular the execution of loops, the latter, as the one presented in this paper, are aimed at optimizing control dominated specifications. Most of the algorithms of the second type work on $C D F G$, but without performing explicit code motion. For example, SDC scheduling algorithm, which has been initially proposed by Cong et al. [2], produces the optimal scheduling of the starting $C D F G$, but it only allows limited implicit code motion (execution of consecutive basic blocks can be only partially overlapped). Explicit code motion is instead considered in [5] where a set of possible code transformations is proposed. Differently from the methodology presented in this paper, the decisions about which are the transformations to be performed are taken on the basis of the results of an heuristic and not of an exact method. The second main difference is that their transformations are aimed at improving only the longest path of the specification and not all the paths at the same time.

The combination of SDC scheduling and code motion has been proposed in [3]. The authors proposed a scheduling framework which performs scheduling of $C D F G$ in two steps. In the former SDC scheduling is used to schedule critical operations after refinement of the $C D F G$. In the latter, after that the scheduling of critical operations has been guaranteed by the insertion of delay operations, a fast heuristic is exploited to schedule the remaining operations allowing their implicit code motion by relaxing conditional constraints. Usage of an heuristic instead of SDC scheduling potentially prevents production of the optimal solution. A quantitative comparison with the approach proposed in this paper has not been possible: the details about experimental setup are not reported in [3] nor it has been possible to compile the Shang framework to generate the data. However, since the authors of Shang framework state that their framework is $30 \%$ faster than LegUp on CHStone benchmarks and since the gain of the proposed methodology with respect to LegUp is much larger (Table I), the results of [3] are expected to be worse than the results of Speculative SDC scheduling. Moreover, the selection of the critical operations is demanded to the designer. Differently from this approach, the methodology proposed in this paper adopts SDC scheduling to compute the schedule of all the operations, potentially allowing code motion of all of them. Moreover, it does not require to introduce delay operations to guarantee the constraints. Finally, SDC algorithm has been extended also to optimize execution of loops. Modulo scheduling and SDC scheduling have been integrated by extending SDC formulation with pipeline dependent constraints and by combining it with greedy heuristic [14] and with backtracking algorithm [15]. In this way it is possible to optimize in an exact way the initiation interval of the loop pipelines and so the overall performance of the applications.

\section{CONClusions AND Future Works}

This paper presents a methodology flow aimed at transforming the $C D F G$ representation of an application to reduce its overall clock cycle latency. The methodology combines Speculative SDC scheduling, global code motion and local heuristics to apply all the profitable transformations. Experimental results show how it is effectively able to improve the performances of hardware implementations of control dominated applications without increasing resource usage. Future works will focus on including profiling information in the proposed Speculative SDC formulation and in allowing code motion inter loops.

\section{REFERENCES}

[1] J. Cong, Bin Liu, S. Neuendorffer, J. Noguera, K. Vissers, and Zhiru Zhang. High-level synthesis for fpgas: From prototyping to deployment. TCAD, 30(4):473-491, April 2011.

[2] Jason Cong and Zhiru Zhang. An efficient and versatile scheduling algorithm based on sdc formulation. DAC '06, pages 433-438, New York, NY, USA, 2006. ACM.

[3] Hongbin Zheng, Qingrui Liu, Junyi Li, Dihu Chen, and Zixin Wang. A gradual scheduling framework for problem size reduction and cross basic block parallelism exploitation in high-level synthesis. In $A S P$ DAC 2013, Yokohama, Japan, January 22-25, 2013, pages 780-786, 2013.

[4] Daniel D. Gajski and Loganath Ramachandran. Introduction to highlevel synthesis. IEEE Des. Test, 11(4):44-54, 1994.

[5] Sumit Gupta, Nick Savoiu, Nikil Dutt, Rajesh Gupta, and Alex Nicolau. Conditional speculation and its effects on performance and area for high-level snthesis. ISSS '01, pages 171-176, New York, NY, USA, 2001. ACM.

[6] Ron Cytron, Jeanne Ferrante, Barry K. Rosen, Mark N. Wegman, and F. Kenneth Zadeck. Efficiently computing static single assignment form and the control dependence graph. ACM TOPLAS, 13(4):451-490, Oct 1991.

[7] R. Cytron, J. Ferrante, and V. Sarkar. Compact representations for control dependence. In PLDI, pages 337-351, 1990.

[8] T. Lengauer and R. Tarjan. A fast algorithm for finding dominators in a flowgraph. (TOPLAS), 1(1):121-141, 1979.

[9] A. Canis, J. Choi, M. Aldham, V. Zhang, A. Kammoona, T. Czajkowski, S. D. Brown, and J.H. Anderson. Legup: An open-source high-level synthesis tool for fpga-based processor/accelerator systems. ACM Trans. Embed. Comput. Syst., 13(2):24:1-24:27, 2013.

[10] Politecnico di Milano. PandA framework. http://panda.dei.polimi.it, 2014.

[11] Xilinx. Vivado Design Suite. http://www.xilinx.com, 2013.

[12] Altera. Quartus II. http://www.altera.com, 2013.

[13] Yuko Hara, Hiroyuki Tomiyama, Shinya Honda, and Hiroaki Takada. Proposal and quantitative analysis of the chstone benchmark program suite for practical c-based high-level synthesis. JIP, 17:242-254, 2009.

[14] Zhiru Zhang and Bin Liu. Sdc-based modulo scheduling for pipeline synthesis. In ICCAD, pages 211-218, Nov 2013.

[15] A. Canis, S. Brown, and J. Anderson. Modulo SDC scheduling with recurrence minimization in high-level synthesis. In FPL, pages 1-8, 2014. 